Case Report

\title{
Gastric Adenocarcinoma Presenting with Gastric Outlet Obstruction in a Child
}

\author{
Abdulrahman Al-Hussaini, ${ }^{1}$ Salem AlGhamdi, ${ }^{1}$ Rasha Alsaaran, ${ }^{2}$ Fawaz Al-Kasim, ${ }^{3}$ \\ Zakaria Habib, ${ }^{4}$ and Nouri Ourfali ${ }^{1}$ \\ ${ }^{1}$ Division of Pediatric Gastroenterology, Hepatology \& Nutrition, Children's Hospital, University of King Saud for Health Sciences, \\ King Fahad Medical City, P.O. Box 59046, Riyadh 11525, Saudi Arabia \\ ${ }^{2}$ Department of Pediatrics, College of Medicine, King Saud University and King Khalid University Hospital, Riyadh, Saudi Arabia \\ ${ }^{3}$ Oncology/Hematology Division, King Saud Medical City, P.O. Box 58594, Riyadh 11515, Saudi Arabia \\ ${ }^{4}$ King Faisal Specialist Hospital and Research Center, Department of Surgery, P.O. Box 3354, Riyadh 11211, Saudi Arabia
}

Correspondence should be addressed to Abdulrahman Al-Hussaini; aa_alhussaini@yahoo.com

Received 7 October 2013; Accepted 17 November 2013; Published 14 January 2014

Academic Editors: S. Hirasaki and F. H. Mourad

Copyright (C) 2014 Abdulrahman Al-Hussaini et al. This is an open access article distributed under the Creative Commons Attribution License, which permits unrestricted use, distribution, and reproduction in any medium, provided the original work is properly cited.

\begin{abstract}
Gastric carcinoma is extremely rare in children representing only $0.05 \%$ of all gastrointestinal malignancies. Here, we report the first pediatric case of gastric cancer presenting with gastric outlet obstruction. Upper endoscopy revealed a markedly thickened antral mucosa occluding the pylorus and a clean base ulcer $1.5 \mathrm{~cm} \times 2 \mathrm{~cm}$ at the lesser curvature of the stomach. The narrowed antrum and pylorus underwent balloon dilation, and biopsy from the antrum showed evidence of Helicobacter pylori gastritis. The biopsy taken from the edge of the gastric ulcer demonstrated signet-ring-cell type infiltrate consistent with gastric adenocarcinoma. At laparotomy, there were metastases to the liver, head of pancreas, and mesenteric lymph nodes. Therefore, the gastric carcinoma was deemed unresectable. The patient died few months after initiation of chemotherapy due to advanced malignancy. In conclusion, this case report underscores the possibility of gastric adenocarcinoma occurring in children and presenting with gastric outlet obstruction.
\end{abstract}

\section{Introduction}

Malignancies of the gastrointestinal tract are very rare in children, accounting for $1.2 \%$ of pediatric malignancies [1]. Malignant gastric tumors that affect children are mostly lymphomas and soft-tissue sarcomas. Gastric carcinomas are extremely rare in children, accounting for $0.05 \%$ of gastrointestinal malignancies [2]. Because of the rarity of gastric carcinoma in children and nonspecificity of presenting symptoms, the diagnosis is usually made late and consequently the prognosis is poor due to established metastases by the time of diagnosis. Here, we report a child with gastric adenocarcinoma and a unique presentation of gastric outlet obstruction. We aim to increase awareness for this type of tumor and from that to improve the prognosis in pediatric patients.

\section{Case Report}

A 10-year-old Saudi girl was referred to our hospital with a complaint of progressive intermittent vomiting for 6 months. The vomiting was projectile of semidigested food content and almost always after meals. It was associated with nonspecific nonradiating epigastric pain, undocumented weight loss, progressive fatigability, and bone aches. There was no history of fever, loose bowel motions, or hematemesis. Her family history was negative for tuberculosis, peptic ulcer diseases, or gastric carcinoma. At physical examination, she was emaciated, sick, pale, and severely dehydrated. There were no palpable pathologic lymph nodes, jaundice, or skin rash. She was afebrile with pulse rate 110 beats/minute, respiratory rate 20 breaths/minute, and blood pressure $90 / 60 \mathrm{mmHg}$. Her weight was $18.9 \mathrm{~kg}(<5$ th percentile for age and sex $)$ and 


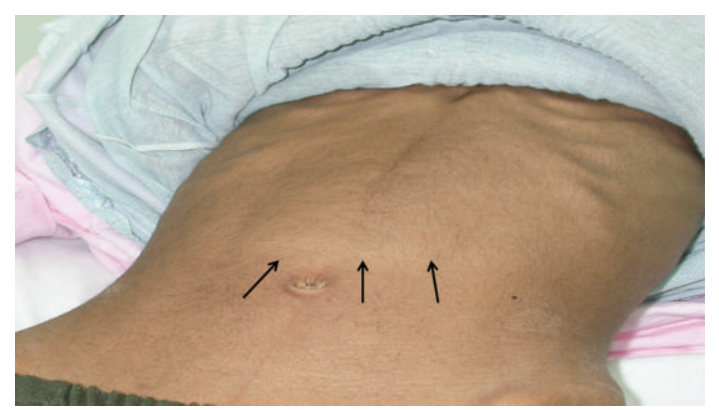

FIgURE 1: Fullness at epigastrium due to gastric distension.

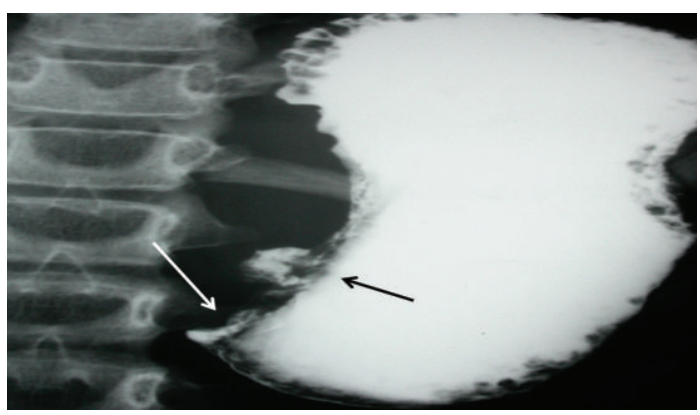

FIGURE 2: Barium meal shows obstruction at pylorus (white arrow) and a crater of an ulcer (black arrow).

height $137.5 \mathrm{~cm}$ (on the 50th percentile). Local examination revealed a soft and lax abdomen with fullness at epigastrium (Figure 1) with no palpable masses, organomegaly, or ascites. Another systemic examination was unremarkable.

Initial laboratory investigations showed hemoglobin $14 \mathrm{gm} / \mathrm{dL}$, white blood cell count $9.2 \times 10^{9} / \mathrm{L}$, platelet count $459 \times 10^{3} / \mathrm{mm}^{3}$, erythrocyte sedimentation rate $30 \mathrm{~mm} / \mathrm{hr}$ (normal, 0-10 mm/hr), and normal peripheral blood smear. Her blood gases revealed $\mathrm{pH} 7.59, \mathrm{PCO}_{2} 50 \mathrm{kPa}$, and $\mathrm{HCO}_{3}$ $55 \mathrm{mmol} / \mathrm{L}$. Other laboratory workup showed hyponatremia $(122 \mathrm{mmol} / \mathrm{L})$, hypokalemia $(1.87 \mathrm{mmol} / \mathrm{L})$, hypochloremia (62 mmol/L), hyperuricemia ( $418 \mathrm{mmol} / \mathrm{L})$, and elevated urea $(13 \mathrm{mmol} / \mathrm{L})$ and creatinine $(69 \mathrm{mmol} / \mathrm{L})$. Her liver function test, serum amylase/lipase and lactate dehydrogenase levels were all normal. At this stage, the clinical diagnosis was gastric outlet obstruction. After correction of dehydration and electrolyte imbalance and establishment of total parenteral nutrition, she underwent barium meal which confirmed the clinical impression of gastric outlet obstruction and in addition showed a crater of an ulcer at lesser curvature (Figure 2).

Ultrasound of abdomen revealed marked thickening of antral wall (12 $\mathrm{mm}$ in diameter) and pyloric channel (Figure 3) and moderate amount of fluid within the pelvis. Upper gastrointestinal endoscopy showed a markedly erythematous, fragile and erosive mucosa at lower esophagus, a markedly thickened antral mucosa occluding the pylorus, and a clean base ulcer 2 centimeters $\times 1.5$ centimeters at the lesser curvature of the stomach (Figure 4). The narrowed

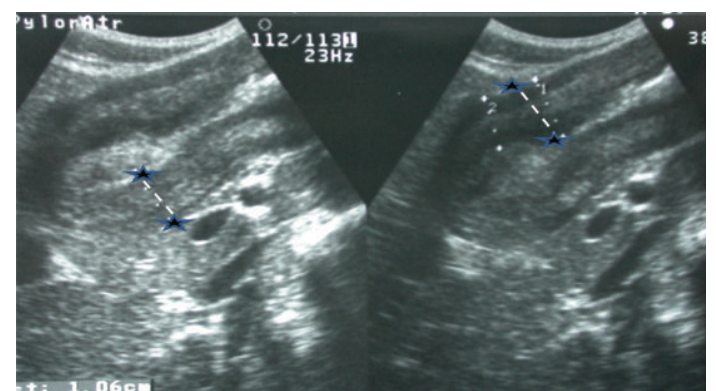

FIGURE 3: Ultrasound abdomen shows thickening of antral (asterisks) and pyloric wall.

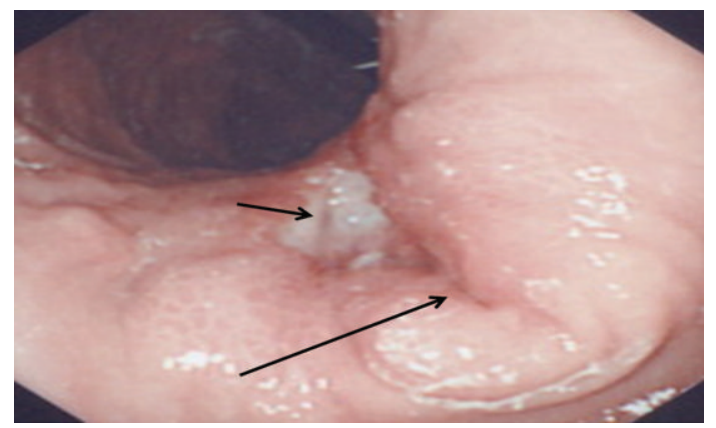

FIGURE 4: Endoscopic view of obstruction at pylorus (long arrow) and a clean bed of an ulcer at lesser curvature (short arrow).

antrum and pylorus underwent balloon dilatation; afterwards, it was possible to pass pediatric size scope $(8.6 \mathrm{~mm})$ through the pylorus down to the duodenum which looked normal and nasojejunal tube was passed through for continuous enteral feeding. The esophageal biopsy showed reflux esophagitis, and the antral biopsies revealed active chronic Helicobacter pylori gastritis grade IV with no metaplastic changes. The biopsies obtained from the edge of the gastric ulcer demonstrated poorly differentiated signet-ring-cell adenocarcinoma (Figure 5). For staging purposes, abdominal CT scan was performed. It revealed marked thickening of antral wall (18 $\mathrm{mm}$ in diameter) and pyloric channel and hypodense soft tissue densities at the celiac axis suggestive of celiac lymphadenopathy, and no definite signs of infiltration either of the liver or pancreas could be visualized. CT scan of the chest showed normal lungs, heart, and pleural spaces and no evidence of metastatic lesions or lymphadenopathy. Bone scan study was negative for evidence of metastases.

The child underwent exploratory laparotomy to evaluate for resectability of the gastric tumor. There were small metastases to the liver, head of pancreas, and mesenteric lymph nodes and aspirate from ascitic fluid revealed malignant cells. Therefore, the gastric carcinoma was deemed unresectable and a gastrojejunostomy tube was placed for enteral nutrition and gastric decompression. The patient received chemotherapy consisting of cisplatin, 5-fluorouracil, and methotrexate. Followup CT scan of abdomen showed no response, so the patient was put on palliative care including analgesics. Unfortunately, she died few months after diagnosis due to advanced carcinoma. 


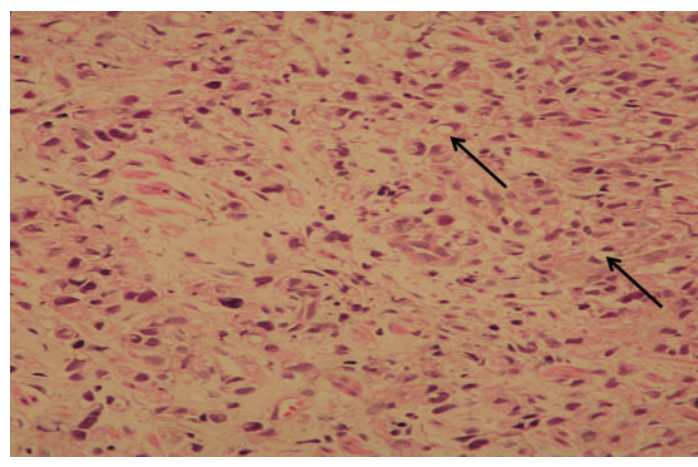

FIGURE 5: Histopathology of a biopsy form edge of the gastric ulcer shows signet-ring-cell infiltrate (arrows) consistent with gastric adenocarcinoma. (Hematoxylin and eosin staining; magnification $\times 40)$.

\section{Discussion}

Gastric carcinoma primarily affects patients in the 50-to-70year age group and is uncommon before the fifth decade of life. Worldwide, there is a large geographic difference in gastric cancer incidence. According to statistics from the International Agency for Research on Cancer, about one million new cases of stomach cancer were estimated to have occurred in 2008 ( $7.8 \%$ of the total), making it currently the fourth most common malignancy in the world, behind cancers of the lung, breast, and colorectum [3]. More than $70 \%$ of cases (713000 cases) occur in developing countries and half the world total occurs in Eastern Asia (mainly in China and Japan). Age-standardized incidence rates (ASR) are about twice as high in men as in women, ranging from 3.9 in Northern Africa to 42.4 in Eastern Asia for men and from 2.2 in Southern Africa to 18.3 in Eastern Asia for women. On the contrary, primary gastric carcinoma is extremely rare in pediatric age group. Our review of medical literature since 1966 revealed that around 30 cases of gastric carcinoma have been reported in children or adolescents.

The 2010 Tumor Registry annual report of the Oncology Center at King Faisal Specialist Hospital and Research Center in Riyadh, Saudi Arabia, showed that a total of 561 cases of gastric cancer were diagnosed over 10-year-period from 2001 to 2010 [4]. Only 2 pediatric cases were diagnosed with gastric cancer during the same period $(0.0035 \%)$.

The rarity of gastric cancer in pediatric age group, unawareness of pediatricians about the possibility of gastric carcinoma in children, and nonspecificity of initial gastrointestinal symptoms led to significant delay in diagnosis and late initiation of appropriate medical therapy and surgical management. Consequently, gastric cancer in children is a more advanced case on presentation and associated with a worse prognosis than adults. The reported 5-year diseasefree survival rate in adults is approximately $15 \%$ [5]. Among the pediatric cases reported in the literature, median survival after diagnosis is approximately 5 months. The only 2 longterm survivors free of disease, 30 and 102 months after surgical resection $[6,7]$, had localized disease that had complete surgical removal of primary tumor. The onset of hematemesis in one case [6] was an alarming symptom that led to early consideration of upper endoscopy and biopsies and early diagnosis of ulcerative gastric cancer.

Symptoms of gastric carcinoma vary according to the location and extent of the tumor, with tumor arising at cardia of the stomach presenting with dysphagia [8] and tumors distal to the cardia are manifesting nonspecific gastrointestinal symptoms like abdominal pain, loss of appetite, weight loss, vomiting, heart burn, anorexia, fatigue, and malaise [6]. Our patient is the first pediatric case to present with gastric outlet obstruction. Other differential diagnoses of gastric outlet obstruction that need to be considered include infectious cause (brucellosis, fungal infection, or tuberculosis), cicatricial gastric ulcer/duodenal ulcer, gastric bezoars, antral web, neoplastic lesions (lymphoma), and inflammatory lesions including Crohn's disease, eosinophilic gastritis, and chronic granulomatous disease [9].

The etiology of gastric cancer has been demonstrated to be multifactorial, infection with Helicobacter pylori being the most important risk factor [10]. Virulence of Helicobacter pylori depends on several factors including the cytotoxic associated gene $\mathrm{A}$ and the vacuolating cytotoxin gene $\mathrm{A}$ [11]. Whether the existence of Helicobacter pylori infection and gastric cancer in our patient is of causal or concurrent relationship remains unclear. Occurrence of gastric cancer in young people motivated molecular studies that identified inherited mutations in the E-cadherin/CDH1 gene described in several ethnic groups [12-14].

In conclusion, gastric carcinoma in children is extremely rare. This case report underscores the possibility of gastric carcinoma occurring in children and should be considered in the differential diagnosis of gastric outlet obstruction. The diagnosis should be made at earlier stages, to provide better chances for these patients to undergo curative treatment.

\section{Conflict of Interests}

The authors declare no conflict of interests.

\section{References}

[1] I. B. Brecht, N. Graf, D. V. Schweinitz, M. C. Frühwald, S. S. Bielack, and D. T. Schneider, "Networking for children and adolescents with very rare tumors: foundation of the gpoh pediatric rare tumor group," Klinische Padiatrie, vol. 221, no. 3, pp. 181-185, 2009.

[2] J. F. Goldthorn and P. C. Canizaro, "Gastrointestinal malignancies in infancy, childhood, and adolescence," Surgical Clinics of North America, vol. 66, no. 4, pp. 845-861, 1986.

[3] International Agency for Research on Cancer (IARC), Globocan 2002, http://globocan.iarc.fr/.

[4] Oncology Center at King Faisal Specialist Hospital and Research Center in Riyadh-Saudi Arabia, "The 2010 tumor registry annual report," http://www.kfshrc.edu.sa/KFCCC/ AnnualReports $/ 2010 \% 20$ Tumor \%20Registry\%20Annual\% 20Report.pdf.

[5] M. T. Harting, M. L. Blakely, C. E. Herzog, K. P. Lally, J. A. Ajani, and R. J. Andrassy, "Treatment issues in pediatric gastric 
adenocarcinoma," Journal of Pediatric Surgery, vol. 39, no. 8, pp. e8-e10, 2004.

[6] T. W. McGill, E. C. Downey, J. Westbrook, D. Wade, and J. de la Garza, "Gastric carcinoma in children," Journal of Pediatric Surgery, vol. 28, no. 12, pp. 1620-1621, 1993.

[7] V. Subbiah, G. Varadhachary, C. E. Herzog, and W. W. Huh, "Gastric adenocarcinoma in children and adolescents," Pediatric Blood and Cancer, vol. 57, no. 3, pp. 524-527, 2011.

[8] B. W. Aichbichler, A. J. Eherer, W. Petritsch, T. A. Hinterleitner, and G. J. Krejs, "Gastric adenocarcinoma mimicking achalasia in a 15-year-old patient: a case report and review of the literature," Journal of Pediatric Gastroenterology and Nutrition, vol. 32, no. 1, pp. 103-106, 2001.

[9] J. P. Otjen, R. S. Iyer, G. S. Phillips, and M. T. Parisi, "Usual and unusual causes of pediatric gastric outlet obstruction," Pediatric Radiology, vol. 42, pp. 728-737, 2012.

[10] C. De Martel and S. Franceschi, "Infections and cancer: established associations and new hypotheses," Critical Reviews in Oncology/Hematology, vol. 70, pp. 183-194, 2009.

[11] A. Kandulski, M. Selgrad, and P. Malfertheiner, "Helicobacter pylori infection: a clinical overview," Digestive and Liver Disease, vol. 40, no. 8, pp. 619-626, 2008.

[12] S. A. Gayther, K. L. Gorringe, S. J. Ramus et al., "Identification of germ-line E-cadherin mutations in gastric cancer families of European origin," Cancer Research, vol. 58, no. 18, pp. 40864089, 1998.

[13] F. M. Richards, S. A. McKee, M. H. Rajpar et al., "Germline Ecadherin gene $(\mathrm{CDH} 1)$ mutations predispose to familial gastric cancer and colorectal cancer," Human Molecular Genetics, vol. 8, no. 4, pp. 607-610, 1999.

[14] S. Iida, Y. Akiyama, W. Ichikawa et al., "Infrequent germ-line mutation of the E-cadherin gene in Japanese familial gastric cancer kindreds," Clinical Cancer Research, vol. 5, no. 6, pp. 1445-1447, 1999. 


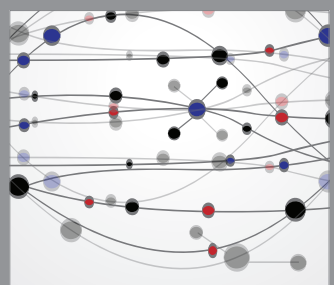

The Scientific World Journal
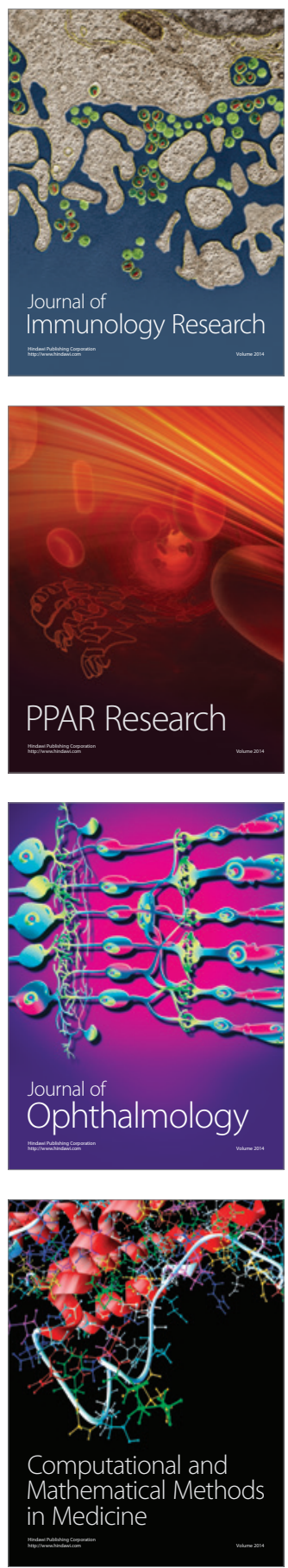

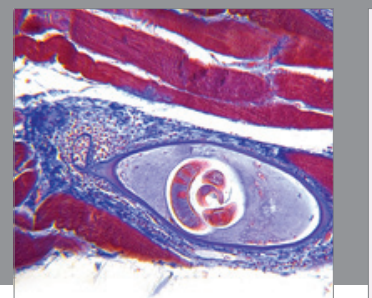

Gastroenterology

Research and Practice
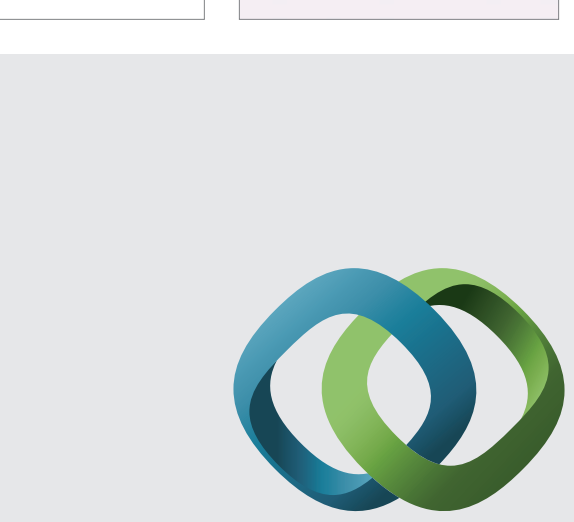

\section{Hindawi}

Submit your manuscripts at

http://www.hindawi.com
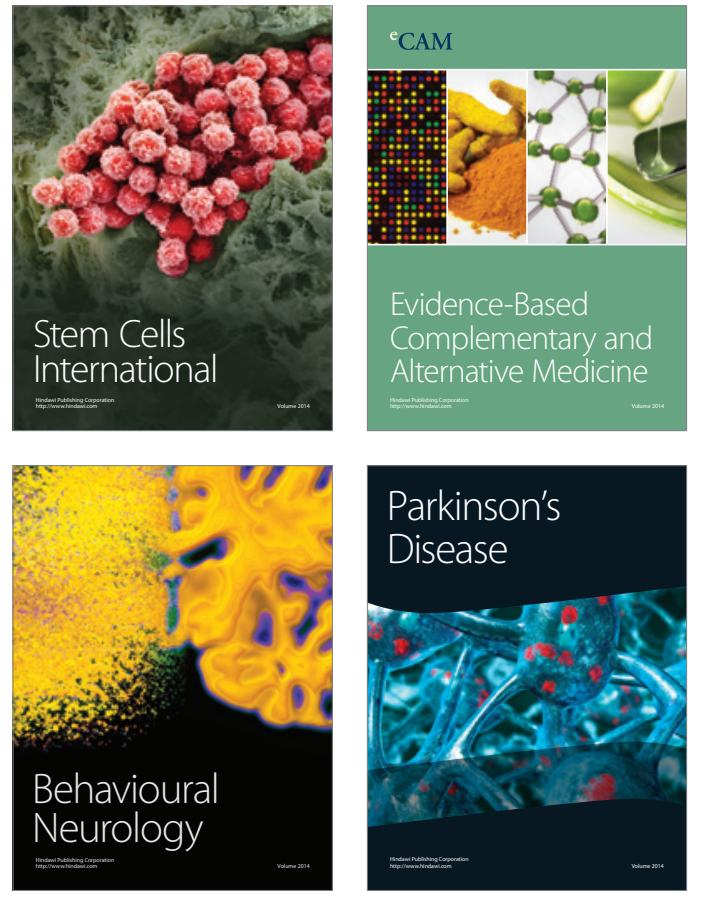
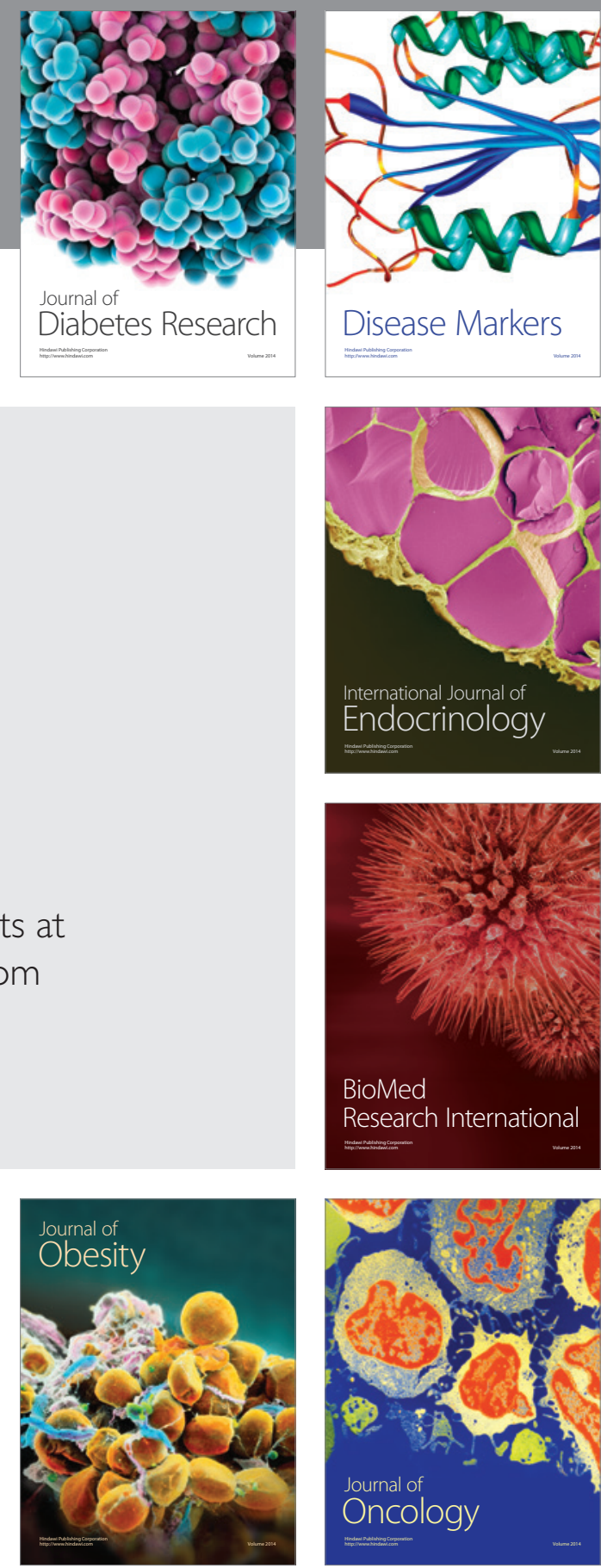

Disease Markers
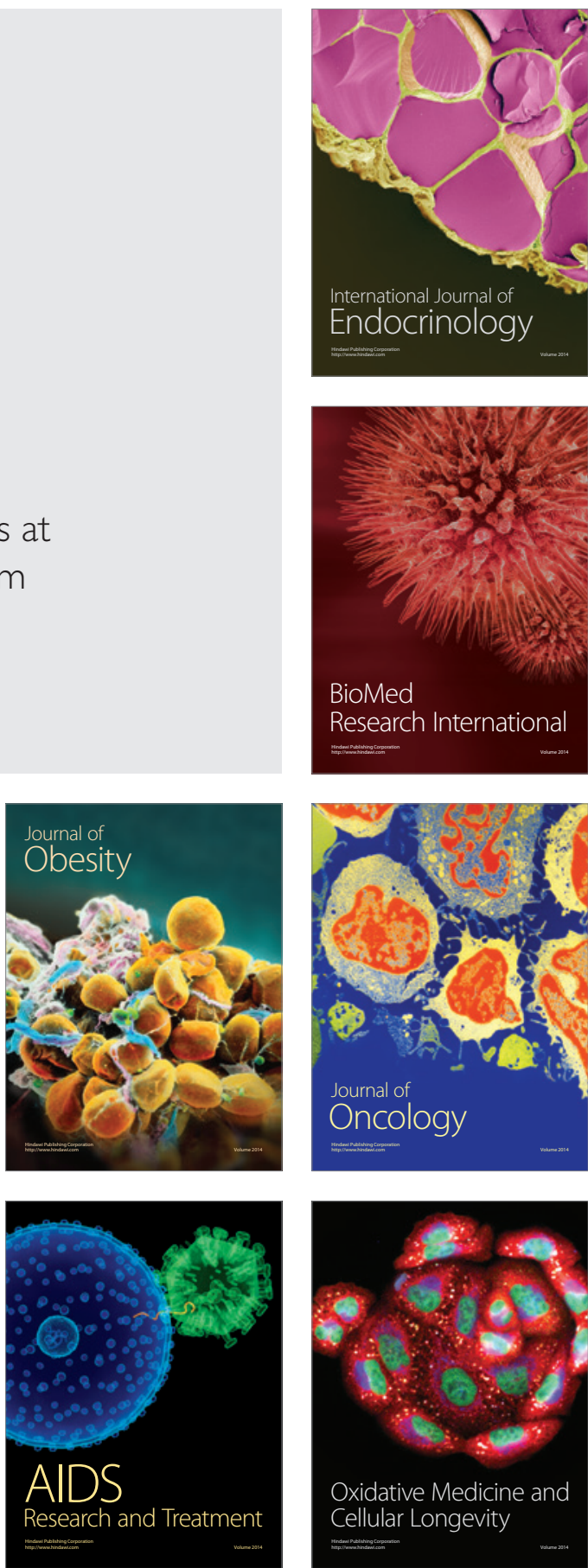